\section{European space science}

SIR - As chairmen of the committees that prepared the Horizon 2000 and the Horizon 2000 Plus long-term programmes for space science within the European Space Agency (ESA), we wish to express our concern about the potential impact of recent proposals, in particular by the United Kingdom, significantly to reduce the funding of ESA's mandatory scientific programme.

Over the past ten years, European space science has reached a high level of achievement in many areas, despite a relatively low level of funding which is, for example, only one-fifth that of projects funded by the US National Aeronautics and Space Administration (NASA).

This has been made possible by a combination of well focused efforts, a carefully planned long-term programme based on the priorities of the scientific community, the timely development of new technologies and a continuous awareness of the need for efficiency and tight cost control.

A significant reduction in the ESA science budget, at a time when the Horizon 2000 programme has developed full momentum, would therefore have a devastating effect on European space science for a number of reasons.

First, the programme will suffer from a disastrous prolongation which is out of proportion to the budgetary decrease. A 10 to 20 per cent decrease, for example, would cause a much greater delay in the execution of the programme, and would lead to the cancellation of some projects on which funds have already been spent, as well as destroying the carefully structured balance between disciplines.

Furthermore, as the Horizon 2000 programme is fully under way, many individual missions are at an advanced stage of development. Prolonging such projects will inevitably lead to substantial increases in the costs incurred by industrial participants, which cannot be retrieved through cost-saving measures because contractual agreements are already in place.

Second, such delays will considerably increase the risk that delays in ESA missions will result in competitors being the first to obtain data of scientific interest. Europe would then return to its secondary role, having abandoned efforts to achieve pre-eminence which have been carefully nurtured over the past decade.

Furthermore, the time gap between successive missions in individual space science disciplines is already considerable. Both the Horizon 2000 and Horizon 2000 Plus programmes were felt to be the minimum needed to sustain the work of leading European space science laboratories and the associated technological developments in European industry.

Further delay will lead to frustration in the space science community, where many scientists have built their careers on the promise of a stable and challenging European space science programme. In addition, creative young scientists and engineers will be discouraged from entering the space field, gravely damaging the long-term prospects for space science.

Third, although economies already achieved by the science programme mean that preparatory activities for Horizon 2000 Plus can be accommodated with present resources, achieving this has not been easy. Budgetary decreases now would mean having to sacrifice future developments in order to salvage as much of the present programme as possible, making the goals of Horizon 2000 Plus impossible to achieve.

For the same reason, existing hardware commitments will put a squeeze on funding to extend missions already in operation. Until now, economies and efficiency gains in the science programme have made it possible to pay for such extensions, sometimes far beyond the original planning period (for example with COS-B and IUE). The science return has in many cases been increased significantly for a relatively small additional investment.

In conclusion, we firmly believe that, without judging whether further economies can be made in ESA programmes in general, no further economies should be made in the resources currently available to the science programme. ESA is in considerable turmoil, and the science programme is one of its few stable elements, acting as the backbone of the whole agency. It is certainly important to seek further improvements in efficiency and cost-effectiveness of the science programme. But the potential savings should remain with the programme.

Ad hoc cuts applied by brute force, and without being based on solid evidence, to a programme in full operation would be utterly irresponsible and eventually counterproductive.

Johan Bleeker

(Chairman Horizon 2000)

SRON - Institute for Space Research,

Sorbonnelaan 2,

3584 CA Utrecht, The Netherlands

\section{Lodewijk Woltjer}

(Chairman Horizon 2000 Plus)

Observatoire de Haut Provence,

F-04870 Saint Michel l'Observatoire,

France

\section{Miracle or fake?}

SIR - You report (Nature 374, 668; 1995) on the case of a figurine of the Virgin Mary that was widely claimed in the Italian media to be weeping "tears of blood".

Is this a miracle? As a scientist and agnostic - despite a Catholic education - I am obviously sceptical. But, as an Italian citizen, I am surprised that a judge, who is an official of the Italian state, should have decided to investigate the authenticity of the so-called miracle, and that he should do so using apparently tough methods.

The judge has "arrested" the figure, even though it had been brought - and not even sold - to a local church by its owner. $\mathrm{He}$ is now attempting not only to submit the tears to chemical analysis, but also to apply DNA tests to the owner of the figurine and his family. As you report, such analysis had already been carried out by scientists at the Policlinico A. Gemelli, a Roman Catholic medical school in Rome, at the request of the Catholic Church. These scientists have already concluded that the tears do indeed contain human blood. But whatever the final explanation, I feel that the only authority that should be investigating the affair is the Catholic Church, with reference to scientific institutions or other consultants if it so chooses.

It is then for the church to decide whether the figure is a 'miracle' or a 'fake' - and for others to accept or reject its conclusions. Bureaucrats and scientists should resist the temptation to impose a reverse inquisition, which would not be compatible with either our constitution or the relationship between the Italian state and the Vatican.

Alfonso M. Liquori

Science Faculty,

University of Rome

"Tor Vergata",

Via della Ricerca Scientifica,

00133 Roma, Italy

\section{Citation analysis}

SIR - Calza and Garbiza (Nature 374, 492; 1995) state: "Computerized analyses of scientific publications allow immediate comparison of applicants by objective parameters", I should like to quote Sydney Brenner (Current Biology 5, 568; 1995): "Before we develop a pseudoscience of citation analysis, we should remind ourselves that what matters absolutely is the scientific content of a paper and that nothing will substitute for either knowing or reading it. We should also recognise that citation often tells us more about sociology of science than about science itself." The assumption that fairness in competitions for university positions can be assured by the proposed allegedly objective criteria is simply ridiculous.

\section{Plergiorgio Strata}

Dipartimento di Anatomia

e Fisiologia Umana,

C.so Raffaello 30 ,

I-10125 Torino, Italy 